TRANSACTIONS OF THE

AMERICAN MATHEMATICAL SOCIETY

Volum 351, Num

S 0002-9947(99)02430-7

Article electronically published on January 27, 1999

\title{
EMBEDDINGS OF OPEN MANIFOLDS
}

\author{
NANCY CARDIM
}

\begin{abstract}
Let TOP $(M)$ be the simplicial group of homeomorphisms of $M$. The following theorems are proved.

Theorem A. Let $M$ be a topological manifold of dim $\geq 5$ with a finite number of tame ends $\varepsilon_{i}, 1 \leq i \leq k$. Let TOP $P^{e p}(M)$ be the simplicial group of end preserving homeomorphisms of $M$. Let $W_{i}$ be a periodic neighborhood of each end in $M$, and let $p_{i}: W_{i} \rightarrow \mathbb{R}$ be manifold approximate fibrations. Then there exists a map $f: T O P^{e p}(M) \rightarrow \prod_{i} T O P^{e p}\left(W_{i}\right)$ such that the homotopy fiber of $f$ is equivalent to $T O P_{c s}(M)$, the simplicial group of homeomorphisms of $M$ which have compact support.

Theorem B. Let $M$ be a compact topological manifold of dim $\geq 5$, with connected boundary $\partial M$, and denote the interior of $M$ by Int $M$. Let $f$ : $\operatorname{TOP}(M) \rightarrow T O P($ Int $M)$ be the restriction map and let $\mathcal{G}$ be the homotopy fiber of $f$ over $i d_{\text {Int } M}$. Then $\pi_{i} \mathcal{G}$ is isomorphic to $\pi_{i} \mathcal{C}(\partial M)$ for $i>0$, where $\mathcal{C}(\partial M)$ is the concordance space of $\partial M$.

Theorem C. Let $q_{0}: W \rightarrow \mathbb{R}$ be a manifold approximate fibration with $\operatorname{dim} W \geq 5$. Then there exist maps $\alpha: \pi_{i} T O P^{e p}(W) \rightarrow \pi_{i} T O P(\hat{W})$ and $\beta: \pi_{i} T \overline{O P}(\hat{W}) \rightarrow \pi_{i} T O P^{e p}(W)$ for $i>1$, such that $\beta \circ \alpha \simeq i d$, where $\hat{W}$ is a compact and connected manifold and $W$ is the infinite cyclic cover of $\hat{W}$.
\end{abstract}

\section{INTRODUCTION}

In this paper we study the homotopy type of the simplicial group of homeomorphisms of an open manifold of dimension $\geq 5$ into itself. There has been extensive research about the homotopy type of $T O P(M)$, for a compact topological manifold $M$. For example, see [4], [9], [38] and the survey papers [10], [11] and [19]. But, if $M$ is a noncompact manifold, very little about this simplicial group is known.

Let $M$ be a topological manifold of $\operatorname{dim} \geq 5$ with a finite number of tame ends $\varepsilon_{i}$, $1 \leq i \leq k$. Each end $\varepsilon_{i}$ of $M$ has a neighborhood $W_{i}$ which is a finitely dominated infinite cyclic cover of a compact and connected manifold. Hughes and Ranicki showed in [13] that for each $W_{i}$, there exists a manifold approximate fibration over $\mathbb{R}, p_{i}: W_{i} \rightarrow \mathbb{R}$. The neighborhood $W_{i}$ is called a periodic neighborhood of $M$.

Denote by $T O P^{e p}(M)$ the simplicial group of end preserving homeomorphisms of $M$. Let $T O P_{c s}(M)$ be the simplicial group of homeomorphisms of $M$ which have compact support. Then $T O P^{e p}(M) \subset T O P(M)$ and $T O P_{c s}(M) \subset T O P^{e p}(M)$.

With those notations, the main result of Section 2 is

Received by the editors November 20, 1996.

1991 Mathematics Subject Classification. Primary 57N37; Secondary 57N35, 57N45.

Key words and phrases. Open manifolds, homeomorphisms of open manifolds, tame ends, manifold approximate fibrations, controlled homeomorphisms.

Partially suported by the CNPq of Brazil. 
Theorem A. There exists a map $f: T O P^{e p}(M) \rightarrow \prod_{i} T O P^{e p}\left(W_{i}\right)$ such that the homotopy fiber of $f$ is equivalent to $T O P_{c s}(M)$.

Hughes' Approximate Isotopy Covering Theorem - Relative Version, and Siebenmann's Recognition Criterion for I-regular neighborhoods have an important role in the proof of this result.

Let $\mathcal{G E}_{\varepsilon_{i}}\left(\mathcal{N}\left(\varepsilon_{i}\right), M\right), 1 \leq i \leq k$, be the simplicial set of equivalence classes of germs of embeddings of a neighborhood of $\varepsilon_{i}$ into $M$ which send $\varepsilon_{i}$ into itself.

The proof of Theorem A is given in two steps. In the first step we show that the map $T O P^{e p}(M) \rightarrow \prod_{i} \mathcal{G E}_{\varepsilon_{i}}\left(\mathcal{N}\left(\varepsilon_{i}\right), M\right)$ is a fibration with fiber $T O P_{c s}(M)$, using Siebenmann's Isotopy Extension Theorem.

In the second step we show that $\prod_{i} T O P^{e p}\left(W_{i}\right) \rightarrow \prod_{i} \mathcal{G E}_{\varepsilon_{i}}\left(\mathcal{N}\left(\varepsilon_{i}\right), M\right)$ is a homotopy equivalence. This homotopy equivalence is a generalization of the Kister-Mazur Theorem: $\operatorname{TOP}\left(\mathbb{R}^{n} ; 0\right) \simeq \mathcal{G} \mathcal{E}_{0}\left(\mathcal{N}(0), \mathbb{R}^{n}\right)$. A new proof of this theorem is given in Section 2, Corollary 2.6.

As an application of Theorem A, a new proof of a theorem of Anderson, Hsiang and Hatcher [3] is given in Section 2, Theorem 2.9.

Kuiper and Lashof in [23] proved a theorem where they express $\operatorname{TOP}\left(\mathbb{R}^{n}\right)$ in terms of $\operatorname{TOP}\left(D^{n}\right)$ and the concordance space for $S^{n-1}, \mathcal{C}\left(S^{n-1}\right)$, i.e.

Kuiper-Lashof Theorem. $\mathcal{C}\left(S^{n-1}\right) \rightarrow T O P\left(D^{n}\right) \rightarrow T O P\left(\mathbb{R}^{n}\right)$ is a homotopy fibration sequence.

In this work, the Kuiper-Lashof Theorem is generalized: $D^{n}$ is replaced by any compact manifold $M$ and $\mathbb{R}^{n}$ by the interior of $M$. That is the main result of Section 3 .

Theorem B. Let $M$ be a compact topological manifold of dim $\geq 5$, with connected boundary $\partial M$, and denote the interior of $M$ by Int $M$. Let $f: \operatorname{TOP}(M) \rightarrow$ $T O P($ Int $M)$ be the restriction map and let $\mathcal{G}$ be the homotopy fiber of $f$ over $i d_{\text {Int } M}$. Then, $\pi_{i} \mathcal{G}$ is isomorphic to $\pi_{i} \mathcal{C}(\partial M)$ for $i>0$, where $\mathcal{C}(\partial M)$ is the concordance space of $\partial M$.

Siebenmann's Isotopy Extension Theorem for CS sets [34] has an important role in the proof of this result.

The map $f$ in Theorem B is not necessarily a fibration, and an example is given of a self-homeomorphism $\rho$ of Int $M$ which is not the restriction of a selfhomeomorphism of $M$ but $\rho$ is isotopic to the identity map.

Finally, in Section 4 we prove

Theorem C. Let $q_{0}: W \rightarrow \mathbb{R}$ be a manifold approximate fibration with dim $W \geq$ 5. Then

1. there exists a manifold approximate fibration $q: \hat{W} \rightarrow S^{1}$ such that the following diagram commutes :

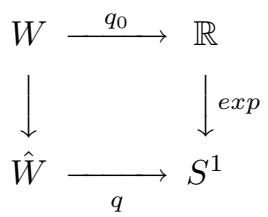

2. $\pi_{n} \operatorname{TOP}^{e p}(W)$ is a direct summand of $\pi_{n} \operatorname{TOP}(\hat{W})$ for $n>1$, where $\hat{W}$ is a compact and connected manifold and $W$ is the infinite cyclic cover of $\hat{W}$. 
The proof of this theorem uses results of Sections 2 and 3.

I would like to thank my advisor, Professor Bruce Williams, for suggesting this work and for his guidance, patience and generous help. My thanks to Bruce Hughes, Steve Ferry, Douglas Anderson, Stratos Prassidis and Mário O. da Silva for their suggestions and useful conversations during the preparation of this paper. Also, I want to thank Larry Taylor, Frank Connolly and Bill Dwyer for making comments for the improvement of this paper.

\section{Preliminaires}

In this section, we establish definitions, results and some properties of the objects that will be used below.

The following definition and examples may be found in Siebenmann [34].

Definition 1.1. A stratified set $X$, in Siebenmann's sense, is a metrizable space $X$ with a filtration $\emptyset=X^{-1} \subset X^{0} \subset \cdots \subset X^{k-1} \subset X^{k} \subset \cdots \subset X$ by closed subsets $X^{k}, k \geq-1$, such that for each $k \geq 0$, the components of $X^{k}-X^{k-1}$ are open in $X^{k}-X^{k-1}$.

It is a top stratified set if $X^{k}-X^{k-1}$ is a topological $k$-manifold without boundary, called the $k$-stratum of $X$.

A stratified set $X$ is locally cone-like if for each $x \in X$, say $x \in X^{k}-X^{k-1}$, there is an open neighborhood $U$ of $x$ in $X^{k}-X^{k-1}$, a compact stratified set of finite dimension $L$ (called a link of $x$ in $X$ ) and a stratum-preserving homeomorphism of $U \times c L$ onto an open neighborhood of $x$ in $X$. (cL is the open cone in $L$. Regard $U$ as a stratified set with $U=U^{k}-U^{k-1}$.)

A $C S$ set is a locally cone-like top stratified set.

Example 1. A topological $m$-manifold $X$ is a CS set. Here $X^{k}=X$ for $k \geq m$, $X^{m-1}=\partial X$, and $X^{i}=\emptyset$ for $i \leq m-2$.

Example 2. Let $M$ be a compact topological manifold with connected boundary $\partial M$. The topological space $X=$ Int $M \cup\{\infty\}$, the one-point compactification of Int $M$, is a CS set. The space $Y=\partial M * S^{0}$, where $\partial M * S^{0}$ denotes the join of $\partial M$ and $S^{0}$, is a CS set.

A mock open cone is a locally compact metric space $C$ with a homotopy $\gamma_{t}$ : $C \rightarrow C$, with $0 \leq t \leq 1$, such that

1. $\gamma_{t}, 0 \leq t<1$, is an isotopy of $i d_{C}$, through homeomorphisms,

2. $\gamma_{0}=i d_{\left.\right|_{C}}, \quad \gamma_{1}(C)=v \in C \quad$ and $\quad \gamma_{t}(v)=v, \forall t$.

A topological stratified set $X$ is a locally weakly cone-like set (WCS) if for each $x \in X^{k}-X^{k-1}$, there is a mock open cone $C$ with vertex $v$ and a homeomorphism $\theta: \mathbb{R}^{k} \times C \rightarrow U$, where $U$ is an open neighborhood of $x$ in $X$, such that $\theta^{-1}\left(X^{k}\right)=$ $\mathbb{R}^{k} \times v$.

Example 3. Open cones on compact sets are trivial examples of mock open cones.

Example 4. Let $W$ be a connected topological manifold of $\operatorname{dim} \geq 5$. Assume $W$ is proper homotopy equivalent to (or even properly dominated by) $F \times \mathbb{R}$, with $F$ a finite connected CW complex. Assume $e_{+}$is one of the two end points of $W$. Then $C=W \cup e_{+}$is a non-trivial example of a mock open cone. A homotopy $\gamma_{t}$ of $W \cup e_{+}$to $e_{+}$can be constructed by an engulfing argument such that (1) and (2) hold and, for each $t, \gamma_{t}$ fixes points outside some compact set in $W$ (depending this time on $t)$. See $[34, \S 5]$. 
Let $M$ be a manifold and $U$ be an open subset of $M$. If $K$ is a subset of $M$ with $K \subset U$, let $\underline{\operatorname{Emb}}(U, M ; K)$ denote the space of proper embeddings of $U$ into $M$ which are the identity on $K$, and let $\underline{\operatorname{Emb}}(U, M)$ denote $\underline{\operatorname{Emb}}(U, M ; \emptyset)$. A neighborhood of $h \in \underline{E m b}(U, M ; K)$ is of the form

$$
N(h)=\{g \in \underline{\operatorname{Emb}}(U, M ; K) / d(g(x), h(x))<\epsilon, \forall x \in C\},
$$

where $C$ is a compact subset of $U, \epsilon>0$ and $d$ is the metric on $M$.

Theorem 1.2 (Deformation Theorem). Let $X$ be a Hausdorff, locally compact, locally connected topological space (CS set or WCS set), $K \subset X$ be a compact set and $V \subset X$ be an open neighborhood of $K$. If $h: V \rightarrow X$ is an open embedding sufficiently near to the inclusion $i: V \hookrightarrow X$ in $\underline{\operatorname{Emb}}(V, X)$, then there exists an isotopy $h_{t}, 0 \leq t \leq 1$, of $h$ through open embeddings $h_{t}: V \rightarrow X$ such that $h_{1}=i$ on $K$ and $h_{t}=h$ outside some compact set in $V$ (independent of $t$ and even of $h)$. Furthermore, the isotopy is standard in the sense that it is constructed to be a continuous function on $h$ as $h$ varies sufficiently near $i$. See [34], and for sufficiently near see [7].

Note. Let $A$ be a subset of a topological space $X$ and $x \in X . A$ is a neighborhood of $x$ if $A$ contains an open set containing $x$.

Lemma 1.3. Let $X$ be a Hausdorff, locally compact, locally connected topological space; let $K$ and $U$ be subsets of $X$ such that $U$ is an open neighborhood of the compact set $K$. Then $K$ has a compact neighborhood $C$ in $X$ such that $C \subset U$.

Proof. Since $X$ is locally compact, $x \in K$ contains a compact neighborhood $C_{x}$ such that $C_{x} \subset U$. Thus, for each $x \in K$ the collection $\mathcal{A}=\left\{\stackrel{\circ}{C}_{x}\right\}_{x \in K}$ is an open cover of $K$. And since $K$ is compact, this implies that there exists a finite subcollection $\left\{\stackrel{\circ}{C}_{x_{1}}, \stackrel{\circ}{C}_{x_{2}}, \ldots, \stackrel{\circ}{C}_{x_{n}}\right\}$ that also covers $K$. Thus, let $C=\bigcup_{i=1}^{n} C_{x_{i}}$ be the compact neighborhood of $K$ in $X$ and $C \subset U$ (since each $C_{x_{i}} \subset U$ ).

Theorem 1.4 (Siebenmann's Isotopy Extension Theorem to $(X, K)$ ). Let $X$ be a Hausdorff, locally compact, locally connected topological space (CS set or WCS set); let $K$ and $V$ be subsets of $X$ such that $V$ is an open neighborhood of the compact set $K$, and such that $K$ has a compact frontier in $V$. Let $f_{t}: V \rightarrow X, t \in I^{n}$, be a continuous family of embeddings, and let $f_{t}(K)$ be closed. Assume that $f_{t}$ respects strata. Then there exists a continuous family of homeomorphisms $F_{t}: X \rightarrow X$, $t \in I^{n}$, fixed outside some compact set, such that $F_{t_{0}}=i d,\left.\quad F_{t}\right|_{K}=f_{t}, \quad \forall t \in I^{n}$ and $F_{t}$ respects strata. See [34, Theorem 6.5].

Remark 1. By Lemma 1.3, the isotopy $F_{t}$ in Siebenmann's Isotopy Extension Theorem above can be chosen such that $F_{t}=f_{t}$ in some compact neighborhood $C$ of $K$ in $X$.

This theorem implies

Theorem 1.5. With the same notation as in Theorem 1.4, the restriction map $T O P(X) \rightarrow \mathcal{G E}(\mathcal{N}(K), X)$ is a (Kan) fibration.

Here $\mathcal{G E}(\mathcal{N}(K), X)$ denotes the simplicial set of embeddings $f: U \times \Delta^{k} \rightarrow X \times \Delta^{k}$ commuting with the projection on $\Delta^{k}$, where $U$ is an open neighborhood of $K$ and two such embeddings $f$ and $f^{\prime}: U^{\prime} \times \Delta^{k} \rightarrow X \times \Delta^{k}$ are identified if they 
agree in a smaller neighborhood of $K$. Let $T O P(X)$ denote the simplicial set of homeomorphisms of $X$. See [5], [24].

Proof. In fact,

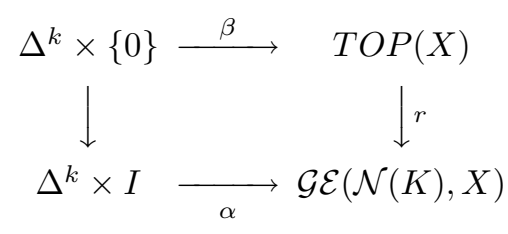

Let $\alpha$ be a $(k+1)$-simplex of $\mathcal{G E}(\mathcal{N}(K), X)$ given by an embedding $q: U \times \Delta^{k} \times$ $I \rightarrow X \times \Delta^{k} \times I$, where $\Delta^{k+1}$ is identified with $\Delta^{k} \times I$ and $U$ is a neighborhood of $K$ in $X$. Let $C \subset U$ be a compact neighborhood of $K$ given by Lemma 1.3. Suppose we are given a lift of the 0 -level of $\alpha$ to a $k$-simplex $\beta$ of $T O P(X)$. Thus $\beta$ is given by the homeomorphism $p: X \times \Delta^{k} \rightarrow X \times \Delta^{k}$ such that $p=q$ on $C \times \Delta^{k}$. Let $i: U \times \Delta^{k} \hookrightarrow X \times \Delta^{k}$ be the inclusion map. Consider the composition $U \times \Delta^{k} \times I \stackrel{q}{\rightarrow} U \times \Delta^{k} \times I \stackrel{i \times i d_{I}}{\longrightarrow} X \times \Delta^{k} \times I$, which is a family of embeddings.

From Theorem 1.4 applied to $(X, C)$, there exists an isotopy of homeomorphisms $f: X \times \Delta^{k} \times I \rightarrow X \times \Delta^{k} \times I$ such that $f=q$ on $C \times \Delta^{k} \times I$ and $\left.f\right|_{X \times \Delta^{k}}=p$. Thus this describes a $(k+1)$-simplex of $\operatorname{TOP}(X)$ which is the required lift of $\alpha$.

Lemma 1.6. Let $X$ and $Y$ be connected Kan simplicial sets with base points $x$ and $y$ respectively. Let $f: X \rightarrow Y$ be a base point preserving map. If $E(f)$, the homotopy fiber of $f$ over $y$, is contractible then $f$ is a homotopy equivalence. For the definition of $E(f)$ see [25].

Lemma 1.7. Let

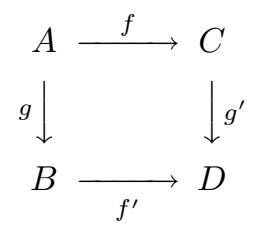

be a commutative diagram of connected based Kan simplicial sets. Then the data determines simplicial maps $\alpha$ and $\beta$ between the homotopy fibers, $\alpha: E(f) \rightarrow E\left(f^{\prime}\right)$ and $\beta: E(g) \rightarrow E\left(g^{\prime}\right)$. Thus, $E(\alpha)$, the homotopy fiber of $\alpha$, is weak homotopy equivalent to the homotopy fiber $E(\beta)$ of $\beta$.

Remark 2. See Adams [1] for the proof of the analogous result for topological spaces.

We refer to Siebenmann's thesis [30] for definition and basic results on ends and tame ends. An end of a manifold is tame if it has a sequence of connected neighborhoods satisfying certain properties. The ends of the interior of a compact manifold are examples of tame ends.

Manifolds with tame ends arise in Siebenmann [31], [33] as finitely dominated infinite cyclic covers of compact manifolds.

Let $X$ be a compact space and $f: X \rightarrow S^{1}$ be a continuous map. Let $Y$ be an infinite cyclic cover of $X$ induced by $f$ from $\exp : \mathbb{R} \rightarrow S^{1}$. Then, there exist a proper map $p: Y \rightarrow \mathbb{R}$ and a generating covering translation $T: Y \rightarrow Y$ such that $p T(y)=p(y)+1, \forall y \in Y$. See [14] and [33]. 
Definition 1.8. A neighborhood $V$ of an end $\varepsilon$ of $M$ is a periodic neighborhood if $V$ is homeomorphic to a finitely dominated infinite cyclic cover of a connected and compact manifold.

Remark 3. Tame ends of an open manifold of dimension $\geq 5$ have periodic neighborhoods. See Siebenmann [31]. This is a special case of the Main Theorem in [18] (see page 1 and let $B=$ point). See also [8].

We now recall some definitions on manifold approximate fibrations. See [14].

Definition 1.9. Let $X$ and $B$ be topological spaces. Given $\epsilon>0$, a map $p: X \rightarrow B$ is an $\epsilon$-fibration if for any space $Z$ and maps $f: Z \rightarrow X, F: Z \times I \rightarrow B$ such that $F(z, 0)=p f(z)$ for $z \in Z$, there exists a map $\tilde{F}: Z \times I \rightarrow X$ such that $\tilde{F}(z, 0)=f(z)$ and $p \tilde{F}$ is $\epsilon$-close to $F$.

An approximate fibration is a map $p: X \rightarrow B$ which is an $\epsilon$-fibration for every $\epsilon>0$.

A manifold approximate fibration is a proper map $p: X \rightarrow B$ which is an approximate fibration and such that $X$ is a finite dimensional manifold without boundary.

The map $p$ in the definition of an approximate fibration is not necessarily onto. But if $p: X \rightarrow B$ is an approximate fibration then the image of $p$ in any path component of $B$ is either empty or dense. In particular, the standard inclusion $(0,1) \hookrightarrow[0,1]$ is an approximate fibration. If $p$ is a closed map then the image of $p$ is closed and hence is either empty or all of a particular path component.

Let $p: X \rightarrow \mathbb{R}$ be a manifold approximate fibration.

Recall from [14] that a $k$-simplex of the simplicial group $T O P^{c}(X \stackrel{p}{\rightarrow} \mathbb{R})$ of controlled homeomorphisms of $X$ is a homeomorphism $h: X \times \Delta^{k} \times[0,1) \rightarrow$ $X \times \Delta^{k} \times[0,1)$ such that $h$ commutes with the projection on $\Delta^{k} \times[0,1)$ and the compositions

$$
X \times \Delta^{k} \times[0,1) \stackrel{h}{\rightarrow} X \times \Delta^{k} \times[0,1) \stackrel{p \times i d}{\longrightarrow} \mathbb{R} \times \Delta^{k} \times[0,1)
$$

and

$$
X \times \Delta^{k} \times[0,1) \stackrel{h^{-1}}{\rightarrow} X \times \Delta^{k} \times[0,1) \stackrel{p \times i d}{\longrightarrow} \mathbb{R} \times \Delta^{k} \times[0,1)
$$

extend continuously to maps

$$
X \times \Delta^{k} \times[0,1] \rightarrow \mathbb{R} \times \Delta^{k} \times[0,1]
$$

via $p \times i d: X \times \Delta^{k} \times[0,1] \rightarrow \mathbb{R} \times \Delta^{k} \times[0,1]$.

Recall from [16] that a $k$-simplex of the simplicial group $T O P^{b}(X \stackrel{p}{\rightarrow} \mathbb{R})$ of bounded homeomorphisms of $X$ consists of a homeomorphism $h: X \times \Delta^{k} \rightarrow X \times \Delta^{k}$ commuting with the projection on $\Delta^{k} \times[0,1)$, and such that $h$ is bounded in the $\mathbb{R}$-direction. Note that a map $f: X \rightarrow Y$ between two topological spaces is called bounded if there exists a number $c>0$, which depends on $f$, such that for each $x \in X,\left\|p_{2} f(x)-p_{1}(x)\right\|<c$, where $p_{1}: X \rightarrow \mathbb{R}$ and $p_{2}: Y \rightarrow \mathbb{R}$.

Remark 4. Hughes and Ranicki in [13, Lemma 7.7] showed that a topological manifold $M$ of dimension $\geq 5$ admits an approximate fibration to $\mathbb{R}$ if and only if $M$ is a finitely dominated infinite cyclic cover of a compact space.

Theorem 1.10. Let $W$ be a connected manifold of $\operatorname{dim} \geq 5$ and let $p: W \rightarrow \mathbb{R}$ be a manifold approximate fibration. Then the following simplicial groups are homotopy equivalent: 
1. $T O P^{e p}(W)$,

2. $T O P^{b}(W \stackrel{p}{\rightarrow} \mathbb{R})$,

3. $T O P^{c}(W \stackrel{p}{\rightarrow} \mathbb{R})$,

where $T O P^{e p}(W)$ denotes the simplicial group of end preserving homeomorphisms of $W$. See [16].

Theorem 1.11. (Hughes'Approximate Isotopy Covering Theorem - Relative Version). Let $p: M \rightarrow B$ be a manifold approximate fibration with $\operatorname{dim} M \geq 5$, and let $B$ be a metric space. Let $C$ and $\tilde{C}$ be closed subsets of $B$ such that $C \subset$ int $\tilde{C}$, let $\alpha$ be an open cover of $B$ and let $h_{t}: B \rightarrow B$ be an isotopy which is supported on $C$. Then there exists an isotopy $H_{t}: M \rightarrow M, 0 \leq t \leq 1$, such that $p H_{t}$ is $\alpha$-close to $h_{t} p$, for each $t$, and $H_{t}$ is supported on $p^{-1}(\tilde{C})$.

Proof. In [16, Theorem 6.1] the case where $C=\emptyset$ is deduced from the Approximation Theorem in [12]. The proof of the relative version is the same except one uses a Relative Approximation Theorem.

We refer to Siebenmann [35] for definitions on I-regular neighborhoods. We summarize the basic results of I-regular neighborhoods. The proofs are essentially in [35], [36], [37].

Let $Y$ be a topological space and $X$ be any subset of $Y$.

I-Compression Axiom. ( $Y, X)$ satisfies I-compression axiom if for any neighborhood $U$ of $X$ in $Y$ there exists a neighborhood $V \subset U$ so that $V$ is I-compressible towards $X$ in $U$.

Remark 5. Under the hypothesis of the I-compression axiom, every regular neighborhood of $X$ in $Y$ is an I-regular neighborhood. See [36, Remark 1.7].

Theorem 1.12 (Uniqueness of I-regular neighborhoods). If $E$ and $E^{\prime}$ are two $I^{-}$ regular neighborhoods of $X$ in $Y$, then there exists an isotopy of embeddings $g_{t}$ : $E \rightarrow Y, 0 \leq t \leq 1$, fixing a neighborhood of $X$ in $Y$ (independent of $t$ ) and such that $g_{0}=\imath$, where $\imath: E \hookrightarrow Y$ is the inclusion and $g_{1}(E)=E^{\prime}$. See [35, Theorem 1.4] or [36, Theorem 2.2].

Theorem 1.13 (Recognition Criterion). Suppose $Y$ is locally compact and $X \subset Y$ is compact. Then an open neighborhood $U$ of $X$ in $Y$ is I-regular if and only if $U$ is $\sigma$-compact, and for each compact set $K \subset U$ there exists a compact set $L \subset U$ such that $K$ is I-compressible towards $X$ in L. See [35, Theorem 3.1] or [36, Theorem 4.1].

Remark 6. Siebenmann in [34, page 254] says that if $(Y, X)$ is compact and metrizable, an open neighborhood $U$ of $X$ is regular if and only if $K$ is compressible towards $X$ in $U$ for each compact set $K \subset U$.

Theorem 1.14. Let $W$ be a topological manifold of dim $\geq 5$, let $\varepsilon$ be an isolated end of $W$ and let $W \cup \varepsilon$ be the one-point compactification of $W$. Suppose that $\varepsilon$ admits I-regular neighborhoods in $(\partial W) \cup \varepsilon$. If $\varepsilon$ is tame then $\varepsilon$ admits I-regular neighborhoods in $W \cup \varepsilon$. See [37, §2].

Remark 7. In the theorem above, if some neighborhood $U$ of $\varepsilon$ is such that $(\partial W) \cap$ $U=\emptyset$, in particular if $\partial W=\emptyset$, then trivially $\varepsilon$ admits I-regular neighborhoods in $(\partial W) \cup \varepsilon$. 
Remark 8. In Theorem 2.7 we will prove that $W \cup e_{+}$is an I-regular neighborhood, for $W$ a total space of a manifold approximate fibration over $\mathbb{R}$.

\section{MANifOLDS With TAME ENDS}

Let $M$ be a non-compact, separable topological manifold of dimension $\geq 5$, with compact (possibly empty) boundary $\partial M$, and let $M$ have a finite number of ends $\varepsilon_{1}, \varepsilon_{2}, \ldots, \varepsilon_{k}$, each one tame. By Remark 3 and Remark 4, for each end $\varepsilon_{i}$ of $M$, choose a periodic neighborhood $W_{i}$ and a manifold approximate fibration $p_{i}: W_{i} \rightarrow \mathbb{R}$.

Let $\operatorname{TOP}(M)$ denote the simplicial group of homeomorphisms of $M$, where a $k$-simplex is a homeomorphism $h: M \times \Delta^{k} \rightarrow M \times \Delta^{k}$ commuting with the projection on $\Delta^{k}$. Let $T O P^{e p}(M)$ denote the simplicial subgroup of $T O P(M)$ of homeomorphisms of $M$ which preserve all the ends of $M$. Notice that $T O P^{e p}(M)$ is the union of certain components of $T O P(M)$.

Let $T O P_{c s}(M)$ be the simplicial subgroup of $T O P^{e p}(M)$ of homeomorphisms of $M$ with compact support.

Let $X$ be a topological space, $K \subset X$ a compact set. Let $\mathcal{G E}_{K}(\mathcal{N}(K), X)$ be the simplicial set of equivalence classes of germs of embeddings whose $k$-simplices are represented by embeddings $h: U \times \Delta^{k} \rightarrow X \times \Delta^{k}$ commuting with the projection on $\Delta^{k}$, for some open neighborhood $U$ of $K$ in $X$ and such that $h(K)=K$. Two such embeddings $h_{i}: U_{i} \times \Delta^{k} \rightarrow X \times \Delta^{k}, i=1,2$, are equivalent if they agree on $U_{3} \times \Delta^{k}$, where $U_{3} \subset U_{1} \cap U_{2}$.

Let $A \subset X$. Let $\operatorname{TOP}(X \mathrm{rel} A)$ denote the simplicial group whose $k$-simplices are homeomorphisms $h: X \times \Delta^{k} \rightarrow X \times \Delta^{k}$ commuting with the projection on $\Delta^{k}$ and which restrict to the identity on $A$.

Theorem A. There exists a map $f: T O P^{e p}(M) \rightarrow \prod_{i} T O P^{e p}\left(W_{i}\right)$ such that the homotopy fiber of $f$ is equivalent to $T O P_{c s}(M) \subset T O P^{e p}(M)$.

Henceforth we shall assume that $M$ has just one tame end $\varepsilon$, with a periodic neighborhood $W$ and a manifold approximate fibration $p: W \rightarrow \mathbb{R}$. Denote by $e_{+}$ and $e_{-}$the two ends of $W$. The general case follows easily.

The main result follows from the analysis of the diagram

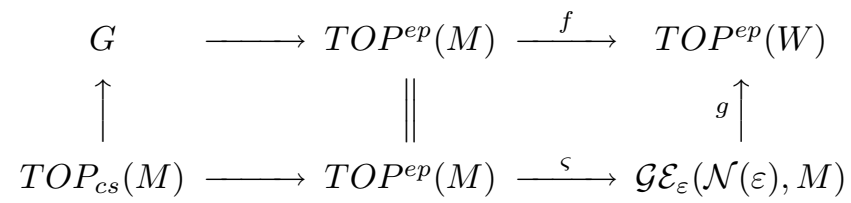

where the following will be proved:

1. The restriction map $\varsigma$ is a fibration with fiber $T O P_{c s}(M)$.

2. The map $g$ is a homotopy equivalence.

In this diagram $G$ denotes the homotopy fiber of $f$ and $\mathcal{G} \mathcal{E}_{\varepsilon}(\mathcal{N}(\varepsilon), M)$ denotes the simplicial set of equivalence classes of germs of embeddings of a neighborhood of $\varepsilon$ into $M$ which send $\varepsilon$ into itself.

The proof of (1) is given in Theorem 2.1.

In order to prove (2) we construct, in Theorem 2.5, a homotopy equivalence $\delta: T O P^{e p}(W) \rightarrow \mathcal{G E}_{\varepsilon}(\mathcal{N}(\varepsilon), M)$. Then let $g$ be a homotopy inverse to $\delta$.

From Theorem 1.10 we have that $\operatorname{TOP}^{e p}(W)$ is homotopy equivalent to $T O P^{c}(W \stackrel{p}{\rightarrow} \mathbb{R})$. 
Proof of Theorem A. Assuming (1) and (2) above, it follows that $G$ is homotopy equivalent to $T O P_{c s}(M)$ in the diagram above, where $f$ is the composition map $f=g \varsigma$.

Let $W \hookrightarrow M$ be a periodic neighborhood of $\varepsilon$. Let $T O P_{W}(M) \subset T O P(M)$ be the subsimplicial group of homeomorphisms of $M$ which restrict to a homeomorphism of $W$.

Corollary A1. (i) $B_{T O P}(M) \rightarrow B T O P(M)$ is a homotopy equivalence.

(ii) $\operatorname{BTOP}_{W}(M) \rightarrow B T O P^{e p}(W)$ is a fibration.

Theorem 2.1. The restriction map $\varsigma: T O P^{e p}(M) \rightarrow \mathcal{G E}_{\varepsilon}(\mathcal{N}(\varepsilon), M)$ is a fibration.

Proof. This follows by applying Theorem 1.5 to $X=M \cup \varepsilon$ and $K=\varepsilon$. Notice that Example 4 (Section 1) of a mock open cone implies that $M \cup \varepsilon$ is a WCS set.

The fiber of $\varsigma$ over the standard embedding is $T O P_{c s}(M)$.

Proposition 2.2. Let $X$ be a topological space, $K \subset X$ a compact set, and let $V$ be an open neighborhood of $K$ in $X$. Then the inclusion $V \subset X$ induces a map $\phi: \mathcal{G E}_{K}(\mathcal{N}(K), V) \rightarrow \mathcal{G E}_{K}(\mathcal{N}(K), X)$ which is a homotopy equivalence.

Proof. Let $h: U \rightarrow V$ be a representative of the class $[h]$ in $\mathcal{G E}_{K}(\mathcal{N}(K), V)$, where $U$ is a neighborhood of $K$ in $V$. Then $i \circ h: U \rightarrow V$ is an embedding such that $i \circ h(K)=K$, where $i$ is the inclusion map. Thus define $\phi: \mathcal{G E}_{K}(\mathcal{N}(K), V) \rightarrow$ $\mathcal{G E}_{K}(\mathcal{N}(K), X)$ by $\phi[h]=[i \circ h]$.

Conversely, let $g: U^{\prime} \rightarrow X$ be an embedding representative of the class $[g]$ in $\mathcal{G E}_{K}(\mathcal{N}(K), X)$, where $U^{\prime}$ is a neighborhood of $K$ in $X$ such that $g(K)=K$. Since $V \subset X$ and $g(K)=K, g^{-1}(V) \supset K$ is an open set. Let $L$ be a neighborhood of $K$ such that $L \subset g^{-1}(V)$. Denote $g^{\prime}=\left.g\right|_{L}$. Then $\bar{g}: L \rightarrow V$ such that $\bar{g}(y)=g^{\prime}(y)$ for $y \in L$ is an embedding in $\mathcal{G E}_{K}(\mathcal{N}(K), V)$. Thus define $\psi: \mathcal{G E}_{K}(\mathcal{N}(K), X) \rightarrow$ $\mathcal{G E}_{K}(\mathcal{N}(K), V)$ by $\psi[g]=[\bar{g}]$.

We have $\phi \circ \psi=i d_{\mathcal{G}_{K}(\mathcal{N}(K), X)}$ and $\psi \circ \phi=i d_{\mathcal{G}_{K}(\mathcal{N}(K), V)}$.

Corollary 2.3. The map $\phi: \mathcal{G E}_{e_{+}}\left(\mathcal{N}\left(e_{+}\right), W\right) \rightarrow \mathcal{G E}_{\varepsilon}(\mathcal{N}(\varepsilon), M)$ is a homotopy equivalence.

Proof. This follows from Proposition 2.2, where $K=e_{+}$which is also the end of $M, V=W$ and $X=M$.

Proposition 2.4. The restriction map $\eta: T O P^{e p}(W) \rightarrow \mathcal{G E}_{e_{+}}\left(\mathcal{N}\left(e_{+}\right), W\right)$ is a homotopy equivalence.

Proof. This is implied by the following claims.

Claim 1. $\eta$ is a fibration.

Proof. This follows from Theorem 2.1 with $M=W$.

The fiber of $\eta$ over the standard embedding is $\operatorname{TOP}\left(W \mathrm{rel} \mathcal{N}\left(e_{+}\right)\right)$.

Claim 2. $\operatorname{TOP}\left(W\right.$ rel $\left.\mathcal{N}\left(e_{+}\right)\right) \simeq *$.

Proof. Let $p: W \rightarrow \mathbb{R}$ be a manifold approximate fibration and $W_{k}=p^{-1}(k,+\infty)$ be a neighborhood of $e_{+}$in $W$. Let $h: W \rightarrow W$ be a homeomorphism such that $\left.h\right|_{W_{k}}=i d$.

Consider a homeomorphism $g: \mathbb{R} \rightarrow \mathbb{R}$ such that $\left.g\right|_{(k,+\infty)}=i d$, where $(k,+\infty)$ is a neighborhood $+\infty$ of in $\mathbb{R}$. 
An isotopy of $g$ to the identity, fixing $(k,+\infty)$, is given by $g_{s}: \mathbb{R} \rightarrow \mathbb{R}, 0 \leq s \leq 1$ :

$$
g_{s}(t)= \begin{cases}g\left(t+\frac{s}{1-s}\right)-\frac{s}{1-s} & \text { if } 0 \leq s<1, \\ i d & \text { if } s=1 .\end{cases}
$$

$g_{s}$ is continuous near 1: given $t \in \mathbb{R}$, choose $s$ close enough to 1 so that $t+\frac{s}{1-s}>$ $k$. Then $g\left(t+\frac{s}{1-s}\right)=t+\frac{s}{1-s}$. Thus, $g_{s}(t)=t+\frac{s}{1-s}-\frac{s}{1-s}=t$.

By Theorem 1.11 there exists a continuous family of homeomorphisms $G_{s}: W \rightarrow$ $W, 0 \leq s \leq 1$, such that $G_{1}=i d$ and $\left(p \times i d_{I}\right) G_{s}$ is close to $g_{s}\left(p \times i d_{I}\right)$. $G_{s}$ is an isotopy of $h$ and the identity, fixing a neighborhood of $e_{+}$contained in $W_{k}$.

Claim 3. $\eta$ is onto on $\pi_{0}$.

Proof. Let $N$ be a neighborhood of $e_{+}$in $W$ such that $N$ is also a total space of a manifold approximate fibration $q: N \rightarrow \mathbb{R}$. Applying Corollary 2.8, there exists an isotopy of embeddings $h_{t}: N \rightarrow W, 0 \leq t \leq 1$, such that $h_{0}=$ inclusion $\imath: N \hookrightarrow W$, $h_{1}=$ homeomorphism, and there exists a smaller neighborhood $V$ of $e_{+}$in $W$ such that $\left.h_{t}\right|_{V}=\left.\imath\right|_{V}$ for all $t$. Let $f: N \rightarrow W$ such that $f\left(e_{+}\right)=e_{+}$be an embedding in $\mathcal{G E}_{e_{+}}\left(\mathcal{N}\left(e_{+}\right), W\right)$. Applying Corollary 2.8 again to $f(N) \subset W$, we get an isotopy of embeddings $g_{t}: f(N) \rightarrow W$ such that $g_{0}=$ inclusion $\imath: f(N) \hookrightarrow W, g_{1}=$ homeomorphism, and there exists a smaller neighborhood $V^{\prime}$ of $e_{+}$in $f(N)$ such that $\left.g_{t}\right|_{V^{\prime}}=\left.g_{0}\right|_{V^{\prime}}$.

Define an isotopy of embeddings $s_{t}: N \rightarrow W, 0 \leq t \leq 1$, by the composition $s_{t}=$ $f g_{t}$ so that $s_{0}=f, s_{1}=$ homeomorphism, and there exists a smaller neighborhood $V^{\prime \prime}$ of $e_{+}$such that $\left.s_{t}\right|_{V^{\prime \prime}}=\left.f\right|_{V^{\prime \prime}}$.

Define $F: W \rightarrow W$ by $F=s_{1}\left(g_{1}\right)^{-1}$. Then $F$ is a homeomorphism such that $\left.F\right|_{V \cap f^{-1}\left(V^{\prime}\right)}=\left.f\right|_{V \cap f^{-1}\left(V^{\prime}\right)}$, i.e., $F$ is a homeomorphism which is germ equivalent to $f$ at $e_{+}$.

Claim 4. Any two fibers of $\eta$ are isomorphic.

Proof. Let $F_{0}=T O P\left(W\right.$ rel $\left.\mathcal{N}\left(e_{+}\right)\right)$be the fiber of $\eta$ over the standard embedding $i: \mathcal{N}\left(e_{+}\right) \rightarrow W$. In particular, $i d_{W} \in F_{0}$. Let $g \in \mathcal{G E}_{e_{+}}\left(\mathcal{N}\left(e_{+}\right), W\right)$ and let $F$ be the fiber of $\eta$ over $g$, i.e., the simplicial group of a homeomorphism $h$ of $W$ into itself such that $\left.h\right|_{\mathcal{N}\left(e_{+}\right)}=g$. Construct an isomorphism $H: F_{0} \rightarrow F$ as follows. Let $h$ be an element in $F$. Define $H: F_{0} \rightarrow F$ by $H(f)=h \circ f$, with $f \in F_{0}$. Since $\left.f\right|_{\mathcal{N}\left(e_{+}\right)}=\left.i d\right|_{\mathcal{N}\left(e_{+}\right)}$, we have that $\left.h \circ f\right|_{\mathcal{N}\left(e_{+}\right)}=\left.h\right|_{\mathcal{N}\left(e_{+}\right)}$. Thus, $H(f)=h \circ f$ is in $F$, i.e. $\eta(h)=\eta(h \circ f)$.

Define the inverse of $H, H_{-1}: F \rightarrow F_{0}$, by $H^{-1}(g)=h^{-1} \circ g$. It is well defined because $h$ is a homeomorphism.

Clearly $H^{-1} \circ H=i d_{F_{0}}$ and $H \circ H^{-1}=i d_{F}$.

Theorem 2.5. The map $\delta: T O P^{e p}(W) \rightarrow \mathcal{G E}_{\varepsilon}(\mathcal{N}(\varepsilon), M)$ is a homotopy equivalence.

Proof. This follows from Corollary 2.3 and Proposition 2.4, where $\delta=\phi \circ \eta$.

As a corollary we have

Corollary 2.6 (Kister - Mazur Theorem). The restriction map $T O P\left(\mathbb{R}^{n} ; 0\right) \rightarrow$ $\mathcal{G E}_{0}\left(\mathcal{N}(0), \mathbb{R}^{n}\right)$ is a homotopy equivalence, where $T O P\left(\mathbb{R}^{n} ; 0\right)$ denotes the simplicial group of homeomorphisms of $\mathbb{R}^{n}$ which fixes the origin.

Proof. In this proof we will use the following claims: 
Claim 1. $T O P^{e p}\left(S^{n-1} \times \mathbb{R}\right) \cong T O P\left(\mathbb{R}^{n} ; 0\right)$.

Proof. Let $h:\left(\mathbb{R}^{n}-0\right) \rightarrow S^{n-1} \times \mathbb{R}$ be a homeomorphism.

Given a homeomorphism $f: \mathbb{R}^{n} \rightarrow \mathbb{R}^{n}$ such that $f(0)=0$, define $\bar{f}: S^{n-1} \times$ $\mathbb{R} \rightarrow S^{n-1} \times \mathbb{R}$ by $\bar{f}=h \circ f \circ h^{-1}$, which is an end preserving homeomorphism. Conversely, given an end preserving homeomorphism $g: S^{n-1} \times \mathbb{R} \rightarrow S^{n-1} \times \mathbb{R}$, define $\bar{g}: \mathbb{R}^{n} \rightarrow \mathbb{R}^{n}$ by

$$
\bar{g}(x)= \begin{cases}h^{-1} \circ g \circ h(x) & \text { for } x \neq 0, \\ 0 & \text { or } x=0 .\end{cases}
$$

Since $g$ is end preserving, $\bar{g}$ is continuous in 0 .

Claim 2. $\mathcal{G E}_{-\infty}\left(\mathcal{N}(-\infty), S^{n-1} \times \mathbb{R}\right) \simeq \mathcal{G} \mathcal{E}_{0}\left(\mathcal{N}(0), \mathbb{R}^{n}\right)$.

Proof. Analogous to Claim 1.

Then, applying Proposition 2.4, where $W=S^{n-1} \times \mathbb{R}$ and $p$ is the projection map, we have that $T O P^{e p}\left(S^{n-1} \times \mathbb{R}\right) \simeq \mathcal{G} \mathcal{E}_{-\infty}\left(\mathcal{N}(-\infty), S^{n-1} \times \mathbb{R}\right)$. And by Claim 1 and Claim 2 we have the corollary.

Theorem 2.7. Let $p: W \rightarrow \mathbb{R}$ be a manifold approximate fibration and let $\operatorname{dim} W \geq 5$. Then $W \cup e_{+}$is an I-regular neighborhood of $e_{+}$.

Proof. It follows from Siebenmann [33] that both of the ends $e_{+}, e_{-}$of $W$ are tame ends. Then, using Theorem 1.14 and Remark 7, we have that $e_{+}$(resp. $\left.e_{-}\right)$ admits I-regular neighborhoods in $W \cup e_{+}$(resp. $\left.W \cup e_{-}\right)$, i.e. $\left(W \cup e_{+}, e_{+}\right)$(resp. $\left.\left(W \cup e_{-}, e_{-}\right)\right)$satisfies the I-compression axiom. Thus, since $\left(W \cup e_{+}, e_{+}\right)$satisfies the I-compression axiom, it follows from Remark 5 that it is enough to show that $W \cup e_{+}$is a regular neighborhood of $e_{+}$. And to show this we apply Remark 6 to $Y=W \cup\left\{e_{+}, e_{-}\right\}, U=W \cup e_{+}$, together with Theorem 1.11.

Let $K=p^{-1}[k, \infty) \cup e_{+}$be a compact set, $K \subset W \cup e_{+}$, and let $V$ be a neighborhood of $e_{+}$. Choose $r$ such that $r>k$ and $p^{-1}[k, \infty) \subset V$.

We will apply Theorem 1.11 to $C=[k-1, r+2], \tilde{C}=[l+1, r+3]$, where $l+1<k-1$, and to the isotopy $h_{t}: \mathbb{R} \rightarrow \mathbb{R}, 0 \leq t \leq 1$, such that $h_{0}=i d$, $h_{1}(x)>r+1$ for $x \geq k$ and $h_{t}$ is supported on $C$. Thus, by Theorem 1.11 there exists an isotopy $H_{t}: W \rightarrow W, 0 \leq t \leq 1$, such that $p H_{t}$ is $\alpha$-close to $h_{t} p$, for each $t$, and $H_{t}$ is supported on $p^{-1}(\tilde{C})$.

Notice the sequence of real numbers $1<l+1<k-1<k<r<r+1<r+2<$ $r+3$.

The isotopy $h_{t}$ of $\mathbb{R}$ is defined by $h_{0}=i d$ and

$$
h_{1}(x)= \begin{cases}x & \text { if } x>r+2 \text { or } x<k-1, \\ x(r-k+2)+(k-1)(k-r-1) & \text { if } k-1 \leq x<k \\ r+1+\frac{x-k}{r-k+2} & \text { if } k \leq x \leq r+2\end{cases}
$$

Since $H_{t}$ is supported on $p^{-1}(\tilde{C}), H$ is the identity on

$$
p^{-1}(-\infty, l+1) \cup p^{-1}(r+3, \infty),
$$

where $p^{-1}(-\infty, l+1) \supset W-p^{-1}[l, \infty)$ and $p^{-1}(r+3, \infty)$ is a neighborhood of $e_{+}$.

Now we verify that $H_{1}(K) \subset V$. Let $x \in K$. Then $p H_{1}(x)$ is $\alpha$-close to $h_{1}(p(x))$. Since $p(x) \geq k$, it follows that $p H_{1}(x) \geq r$ (because $h_{1}(p(x)) \geq r+1$ by the construction of $\left.h_{1}\right)$. It means that $H_{1}(x) \subset p^{-1}([r, \infty)) \subset V$. 
Since $H_{t}$ is fixed on a neighborhood of $e_{+}$, we can extend $H_{t}$ to $\bar{H}_{t}: W \cup e_{+} \rightarrow$ $W \cup e_{+}$by $\left.\bar{H}_{t}\right|_{W}=H_{t}$ and $\bar{H}_{t}\left(e_{+}\right)=e_{+}$. Thus, $K$ is compressible towards $e_{+}$in $U$.

Corollary 2.8. Let $p: W \rightarrow \mathbb{R}$ be a manifold approximate fibration and suppose that $U$ is an open neighborhood of $e_{+}$in $W$ such that $U$ is also the total space of a manifold approximate fibration $q: U \rightarrow \mathbb{R}$. Then there exists an isotopy of embeddings $h_{t}: U \rightarrow W, 0 \leq t \leq 1$, such that, $h_{0}=\imath$, where $\imath$ is the inclusion map $\imath: U \hookrightarrow W, h_{1}$ is a homeomorphism and $h_{t}$ fixes a smaller neighborhood $V$ of $e_{+}$.

Proof. This follows from Theorem 1.12, where $E=U \cup e_{+}$and $E^{\prime}=W \cup e_{+}$are I-regular neighborhoods.

We now use Theorem 2.1 to give an alternative proof of Anderson and Hsiang's Theorem [3] as given in the next theorem.

Let $N$ be a compact, connected manifold and let $p: N \times \mathbb{R} \rightarrow \mathbb{R}$ be the projection map.

Theorem 2.9 (Anderson-Hsiang-Hatcher). $\Omega\left(T O P^{b}(N \times \mathbb{R})\right) \simeq T O P(N \times I$ rel $\partial)$.

Proof. Fact $\left(^{*}\right)$ : If $X$ is a topological space, $x \in X$ is a base point, and $\Delta: X \rightarrow$ $X \times X$ is the diagonal map, then the homotopy fiber of $\Delta$ at $(x, x)$ is homotopy equivalent to $\Omega(X, x)$.

This fact will be used in the proof of this theorem.

From Theorem 2.1 applied to $M=N \times \mathbb{R}$ we have that the restriction maps

$$
\mu_{+}: T O^{e p}(N \times \mathbb{R}) \rightarrow \mathcal{G} \mathcal{E}_{+\infty}(\mathcal{N}(+\infty), N \times \mathbb{R})
$$

and

$$
\mu_{-}: T O P^{e p}(N \times \mathbb{R}) \rightarrow \mathcal{G} \mathcal{E}_{-\infty}(\mathcal{N}(-\infty), N \times \mathbb{R})
$$

are fibrations. The homotopy fiber of the map

$$
\Phi: T O P^{e p}(N \times \mathbb{R}) \rightarrow \mathcal{G} \mathcal{E}_{+\infty}(\mathcal{N}(+\infty), N \times \mathbb{R}) \times \mathcal{G E}_{-\infty}(\mathcal{N}(-\infty), N \times \mathbb{R})
$$

is

$$
\operatorname{TOP}(N \times \mathbb{R} \operatorname{rel}\{\mathcal{N}(+\infty), \mathcal{N}(-\infty)\})
$$

which is homotopy equivalent to $T O P(N \times I$ rel $\partial)$.

So, we construct the following diagram:

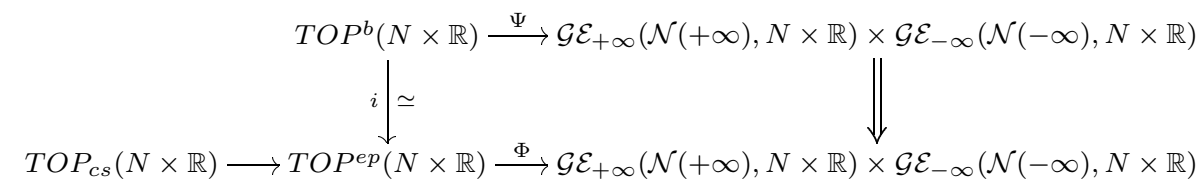

where $\Psi$ is the composition $\Psi=\Phi \circ i$ with $i$ a homotopy equivalence. See Theorem 1.10 .

Then, the homotopy fiber of the map $\Psi$ is equivalent to the fiber of $\Phi$, which is homotopy equivalent to $\operatorname{TOP}(N \times I$ rel $\partial)$. Finally, by fact $(*)$, the homotopy fiber of $\Phi$ at (incl, incl) is equivalent to $\Omega\left(T O P^{b}(N \times \mathbb{R})\right)$. In other words, $T O P(N \times I$ rel $\partial)$ is homotopy equivalent to $\Omega\left(T O P^{b}(N \times \mathbb{R})\right)$. 


\section{MANifolds Which ARE THE INTERIOR OF A COMPACT MANIFOLD}

In this section, a generalization of the Kuiper-Lashof Theorem is given for a non-compact manifold which is the interior of a compact manifold with connected boundary.

Through this section all embeddings are proper.

Let $M$ be a compact topological manifold of dimension $\geq 5$, with connected boundary $\partial M$, and denote the interior of $M$ by Int $M$.

Let $\mathcal{C}(\partial M)$ denote the space of concordances of $\partial M$.

Let $f: T O P(M) \rightarrow T O P($ Int $M)$ be the restriction map, and let $\mathcal{G}$ be the homotopy fiber of $f$ over $i d_{\text {Int } M}$.

Theorem B. $\pi_{i} \mathcal{G}$ is isomorphic to $\pi_{i} \mathcal{C}(\partial M)$, for $i>0$.

This result follows from the of the diagram

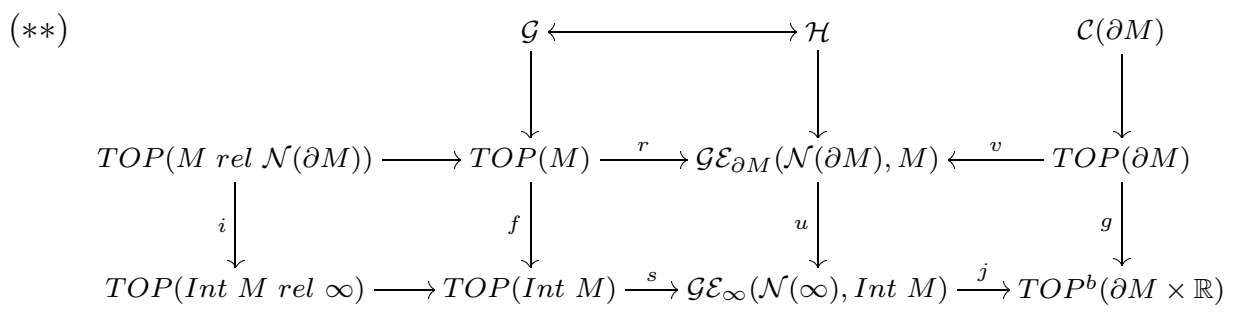

where the following will be proved:

(1) the restriction maps $r$ and $s$ are fibrations, with fibers $\operatorname{TOP}(M \operatorname{rel} \mathcal{N}(\partial M))$ and $T O P($ Int $M$ rel $\infty)$;

(2) the maps $j$ and $v$ are homotopy equivalences;

(3) the diagrams (I)

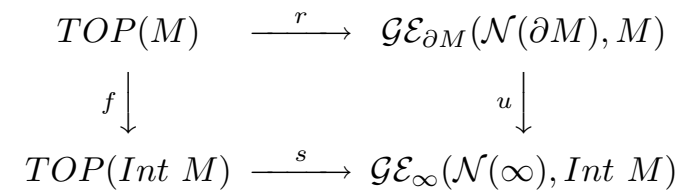

and (II)

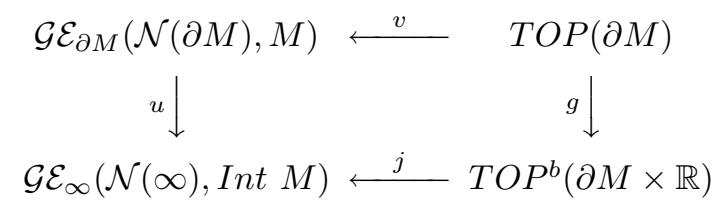

are commutative.

The proof of (1) will be given in Theorems 3.1 and 3.2. The maps $j$ and $v$ will be constructed in Theorems 3.5 and 3.10. The construction depends on the choice of a collar for $\partial M$. In Remarks 3.6 and 3.11 we have (3).

It was proved by Anderson and Hsiang [3] that $\mathcal{C}(\partial M)$ is the homotopy fiber of the map $g=-\times i d_{\mathbb{R}}: T O P(\partial M) \rightarrow T O P^{b}(\partial M \times \mathbb{R})$.

Proof of Theorem B. Let $\mathcal{H}$ denote the homotopy fiber of $u$. Assume (1) $-(3)$ above. Then:

1. $i: \operatorname{TOP}(M$ rel $\mathcal{N}(\partial M)) \rightarrow T O P($ Int $M$ rel $\infty)$ is an isomorphism (Remark $3.12)$.

2. Lemma 1.7 applied to the square (I) implies that $\pi_{i} \mathcal{G} \cong \pi_{i} \mathcal{H}$, for $i>0$. 
3. Since the square (II) commutes, and $j$ and $v$ are homotopy equivalences, and we get that $\pi_{i} \mathcal{H} \cong \pi_{i} \mathcal{C}(\partial M)$, for $i>0$.

Thus, $\pi_{i} \mathcal{G} \cong \pi_{i} \mathcal{C}(\partial M)$, for $i>0$.

The technique used cannot be applied for the case $i=0$, because it works only for connected sets. See Lemma 1.7 and Remark 2.

Theorem 3.1. The restriction map $r: T O P(M) \rightarrow \mathcal{G E}_{\partial M}(\mathcal{N}(\partial M), M)$ is a fibration.

Proof. This follows from Theorem 1.5. The fiber of $r$ over the inclusion map is $\operatorname{TOP}(M \operatorname{rel} \mathcal{N}(\partial M))$.

Theorem 3.2. The restriction map $s: T O P($ Int $M) \rightarrow \mathcal{G E}_{\infty}(\mathcal{N}(\infty)$, Int $M)$ is a fibration.

Proof. This is a special case of Theorem 2.1, where $M=$ Int $M$. The fiber of $s$ is TOP $($ Int $M$ rel $\infty)$.

The homotopy equivalence $j: T O P^{b}(\partial M \times \mathbb{R}) \rightarrow \mathcal{G} \mathcal{E}_{\infty}(\mathcal{N}(\infty)$, Int $M)$ is based on Lemmas 3.3 and 3.4, and on a choice of a collar for $\partial M$ in $M$.

Choose a collar $c: \partial M \times[0,1) \rightarrow M$ for $\partial M$ in $M . c$ induces an isomorphism of simplicial sets.

Lemma 3.3. $i_{c}: \mathcal{G E}_{+\infty}(\mathcal{N}(+\infty), \partial M \times \mathbb{R}) \rightarrow \mathcal{G E}_{\infty}(\mathcal{N}(\infty)$, Int $M)$ is a homotopy equivalence.

Proof. With the above choice of a collar $c$, this follows from Corollary 2.3 with $W=\partial M \times \mathbb{R}$.

Lemma 3.4. The restriction map $\mu: T O P^{b}(\partial M \times \mathbb{R}) \rightarrow \mathcal{G} \mathcal{E}_{+\infty}(\mathcal{N}(+\infty), \partial M \times \mathbb{R})$ is a homotopy equivalence.

Proof. This follows from Theorem 1.10 and Proposition 2.4 for the special case where $W=\partial M \times \mathbb{R}$ and $p: \partial M \times \mathbb{R} \rightarrow \mathbb{R}$ is the projection map.

Theorem 3.5. The map $j: T O P^{b}(\partial M \times \mathbb{R}) \rightarrow \mathcal{G E}_{\infty}(\mathcal{N}(\infty)$, Int $M)$ is a homotopy equivalence.

Proof. The proof follows from Lemmas 3.3 and 3.4 as indicated in the diagram

$$
T O P^{b}(\partial M \times \mathbb{R}) \underset{\sim}{\stackrel{3.4}{\sim}} \mathcal{G} \mathcal{E}_{+\infty}(\mathcal{N}(+\infty), \partial M \times \mathbb{R}) \underset{i_{c}}{\stackrel{3.3}{\sim}} \mathcal{G} \mathcal{E}_{\infty}(\mathcal{N}(\infty), \text { Int } M) .
$$

Thus $j=\mu \circ i_{c}$.

Remark 3.6. The commutativity of square I follows by inspectiom since the maps $r, s, u$ and $f$ are all restriction maps.

Now, with the same choice of the collar $c$ we will construct the homotopy equivalence $v$. This construction is based on Lemma 3.7 through Proposition 3.9. For this, we define maps $\alpha, \beta, \gamma, v$ and $k$ such that $k=\alpha \circ \gamma^{-1}$ and $v=\gamma \circ \beta$, as follows.

The map $\gamma: \mathcal{G E}_{\partial M}(\mathcal{N}(\partial M), \partial M \times[0,1)) \rightarrow \mathcal{G E}_{\partial M}(\mathcal{N}(\partial M), M)$ is defined in terms of the collar $c$, and it is a homotopy equivalence.

The map $\alpha: \mathcal{G E}_{\partial M}(\mathcal{N}(\partial M), \partial M \times[0,1)) \rightarrow T O P(\partial M)$ is defined as the restriction map and we will show that it is a homotopy equivalence. 
The map $\beta: T O P(\partial M) \rightarrow \mathcal{G} \mathcal{E}_{\partial M}(\mathcal{N}(\partial M), \partial M \times[0,1))$ is defined as $\beta=-\times$ $i d_{[0,1)}$, and it is a homotopy equivalence. Thus the map $k: \mathcal{G E}_{\partial M}(\mathcal{N}(\partial M), M) \rightarrow$ $T O P(\partial M)$, defined as the restriction map, is a homotopy equivalence, and the map $v: T O P(\partial M) \rightarrow \mathcal{G E}_{\partial M}(\mathcal{N}(\partial M), M)$ defined by $v=\gamma \circ \beta$ is a homotopy equivalence.

Lemma 3.7. The map $\gamma: \mathcal{G E}_{\partial M}(\mathcal{N}(\partial M), \partial M \times[0,1)) \rightarrow \mathcal{G E}_{\partial M}(\mathcal{N}(\partial M), M)$ is a homotopy equivalence.

Proof. This follows from Corollary 2.3.

Proposition 3.8. The restriction map $\alpha: \mathcal{G E}_{\partial M}(\mathcal{N}(\partial M), \partial M \times[0,1)) \rightarrow T O P(\partial M)$ is a homotopy equivalence.

Proof. The Isotopy Extension Theorem for topological manifolds [6, Corollary 1.4] applied here implies by Theorem 1.5 that $\alpha$ is a (Kan) fibration, and it is surjective. The fiber of $\alpha$ over the identity map is $\mathcal{G E}(\mathcal{N}(\partial M), \partial M \times[0,1)$; rel $\partial M)$, the simplicial set of equivalence classes of germs of embeddings from a neighborhood of $\partial M$ to $\partial M \times[0,1)$ which restrict to the identity on $\partial M$.

We will show that $\pi_{i} \mathcal{G E}(\mathcal{N}(\partial M), \partial M \times[0,1)$; rel $\partial M)$ is trivial for all $i$.

For any $0<a<1$, we have a map

$$
\begin{aligned}
r_{a}: E m b & (\partial M \times[0, a), \partial M \times[0,1) ; \text { rel }(\partial M \times 0)) \\
\rightarrow & \mathcal{G E}(\mathcal{N}(\partial M), \partial M \times[0,1) ; \text { rel } \partial M)
\end{aligned}
$$

which sends each embedding into its class of germ.

We will prove the following two facts.

(1) Given any map $\lambda: S^{n} \rightarrow \mathcal{G E}(\mathcal{N}(\partial M), \partial M \times[0,1)$; rel $\partial M)$, there exist a number $a_{0}$ (which we will denote simply by $a$ ) and a map

$$
\bar{\lambda}: S^{n} \rightarrow \operatorname{Emb}(\partial M \times[0, a), \partial M \times[0,1) ; \operatorname{rel}(\partial M \times 0))
$$

such that $r_{a} \circ \bar{\lambda} \simeq \lambda$ and

(2) Given any $\bar{\lambda}: S^{n} \rightarrow E m b(\partial M \times[0, a), \partial M \times[0,1)$; rel $(\partial M \times 0))$, there exist $h_{s}: S^{n} \rightarrow E m b(\partial M \times[0, a), \partial M \times[0,1) ;$ rel $\mathcal{N}(\partial M \times 0)), 0 \leq s<\infty$, such that $h_{0}=\bar{\lambda}$ and for all $s>0, h_{s} \in E m b(\partial M \times[0, a), \partial M \times[0,1) ; r e l(\partial M \times 0))$.

Proof of item (1). Let $\lambda: S^{n} \rightarrow \mathcal{G E}(\mathcal{N}(\partial M), \partial M \times[0,1)$; rel $\partial M)$ be a continuous map. For each $z \in S^{n}$, let $b_{z}: \partial M \times\left[0, a_{z}\right) \rightarrow \partial M \times[0,1)$ be a representative of the class $\lambda(z) \in \mathcal{G E}(\mathcal{N}(\partial M), \partial M \times[0,1) ;$ rel $\partial M)$, where $\partial M \times\left[0, a_{z}\right)$ is a neighborhood of $\partial M$ in $\partial M \times[0,1)$. By continuity of $\lambda, b_{z}$ is such that the map $S^{n} \rightarrow(0,1)$; $z \mapsto a_{z}$ is continuous, and since $S^{n}$ is compact, $b_{z}$ has a minimum value, say $a>0$. Then $b_{z_{1}}: \partial M \times[0, a) \rightarrow \partial M \times[0,1)$ still is the same class $\lambda(z)$. Then consider $\bar{\lambda}: S^{n} \rightarrow \operatorname{Emb}(\partial M \times[0, a), \partial M \times[0,1)$; rel $(\partial M \times 0))$ such that $z \mapsto b_{z}$ and $r_{a}: \operatorname{Emb}(\partial M \times[0, a), \partial M \times[0,1) ; \operatorname{rel}(\partial M \times 0)) \rightarrow \mathcal{G E}(\mathcal{N}(\partial M), \partial M \times[0,1) ;$ rel $\partial M)$ which sends each embedding into its class of germ such that $r_{a} \circ \bar{\lambda} \simeq \lambda$.

Proof of item (2). Let $f: \partial M \times[0, a) \rightarrow \partial M \times[0,1)$ be an embedding such that $\left.f\right|_{\partial M \times 0}=i d$. We define an isotopy $h_{s}: \partial M \times[0, a) \rightarrow \partial M \times[0,1)$ in the following way. 
Set $I_{s}=[-s, a)$, for $s \in[0, \infty)$. First, define an auxiliary family of embeddings $f_{s}: \partial M \times I_{s} \rightarrow \partial M \times I_{s}$ by

$$
f_{s}(x, t)= \begin{cases}(x, t) & \text { if } t \in[-s, 0] \\ f(x, t) & \text { if } t \in[0, a)\end{cases}
$$

Since $\left.f\right|_{\partial M \times 0}=i d, f_{s}$ is well defined, it is continuous, and it is an embedding $\forall s \in[0,1]$. Also, $f_{0}=f$.

Now, for each $s \in[0, \infty)$ consider the homeomorphisms $g_{s}:[-s, a) \rightarrow[0, a)$ defined by $g_{s}(t)=\frac{a(t+s)}{a+s}$. Notice that $g_{0}=i d_{[0, a)}$.

Finally define an isotopy $h_{s}: \partial M \times[0, a) \rightarrow \partial M \times[0,1)$ by

$$
h_{s}(x, t)=\left(i d_{\partial M} \times g_{s}\right) \circ f_{s} \circ\left(i d_{\partial M} \times\left(g_{s}^{-1}\right)(x, t)=\left(i d_{\partial M} \times g_{s}\right) \circ f_{s}\left(x,\left(g_{s}^{-1}\right)(t)\right)\right. \text {. }
$$

We have $h_{0}=f_{0}=f$, and for $t \in\left[0, \frac{s a}{s+a}\right]$ we have $\left(g_{s}\right)^{-1}(t) \leq 0$, which implies $f_{s}\left(x,\left(g_{s}\right)^{-1}(t)\right)=\left(x,\left(g_{s}\right)^{-1}(t)\right)$. Thus, for $t \in\left[0, \frac{s a}{s+a}\right]$,

$$
h_{s}(x, t)=\left(i d_{\partial M} \times g_{s}\right) \circ f_{s}\left(x,\left(g_{s}\right)^{-1}(t)\right)=\left(i d_{\partial M} \times g_{s}\right)\left(x,\left(g_{s}\right)^{-1}(t)\right)=(x, t) .
$$

This shows that $\pi_{0} E m b(\partial M \times[0, a), \partial M \times[0,1) ;$ rel $\partial M)=0$.

Analogously, for $i \geq 1, \pi_{i} E m b(\partial M \times[0, a), \partial M \times[0,1)$; rel $\partial M)=0$. $i d$.

Consider $f: S^{n} \times \partial M \times[0, a) \rightarrow \partial M \times[0,1)$ such that for each $z \in S^{n},\left.f\right|_{\partial M \times 0}=$

Set $I_{s}=[-s, a)$, for $s \in[0, \infty)$. Define an auxiliary family of embeddings $f_{s}: S^{n} \times \partial M \times I_{s} \rightarrow \partial M \times I_{s}$ by

$$
f_{s}(z, x, t)= \begin{cases}(x, t) & \text { if } t \in[-s, 0], \\ f(z, x, t) & \text { if } t \in[0, a) .\end{cases}
$$

And $f_{s}$ has the same properties as before.

Consider the same family of homeomorphisms $g_{s}$. Then define an isotopy $h_{s}$ : $S^{n} \times \partial M \times[0, a) \rightarrow \partial M \times[0,1)$ by

$$
\begin{aligned}
h_{s}(z, x, t) & =\left(i d_{\partial M} \times g_{s}\right) \circ f_{s} \circ\left(i d_{S^{n}} \times i d_{\partial M} \times\left(g_{s}^{-1}\right)(z, x, t)\right. \\
& =\left(i d_{\partial M} \times g_{s}\right) \circ f_{s}\left(z, x,\left(g_{s}^{-1}\right)(t)\right) .
\end{aligned}
$$

If we apply the map $r_{a}$ to this homotopy, we then get a homotopy in $\mathcal{G E}(\mathcal{N}(\partial M), \partial M \times[0,1) ;$ rel $\partial M)$ such that $r_{a} \circ h_{0}=\lambda$ and $\forall s>0, r_{a} \circ h_{s} \in$ $\mathcal{G E}(\mathcal{N}(\partial M), \partial M \times[0,1) ;$ rel $\partial M)$.

Proposition 3.9. The restriction map $k: \mathcal{G E}_{\partial M}(\mathcal{N}(\partial M), M) \rightarrow T O P(\partial M)$ is a homotopy equivalence.

Proof. The map $k=\alpha \circ \gamma^{-1}$ is indicated in the following diagram:

$$
\mathcal{G} \mathcal{E}_{\partial M}(\mathcal{N}(\partial M), M) \stackrel{\gamma^{-1}}{\longrightarrow} \mathcal{G} \mathcal{E}_{\partial M}(\mathcal{N}(\partial M), \partial M \times[0,1)) \stackrel{\alpha}{\longrightarrow} T O P(\partial M),
$$

where $\gamma^{-1}$ and $\alpha$ are homotopy equivalences, which are proved in Lemma 3.7 and 3.8. So, $k$ is a homotopy equivalence.

Theorem 3.10. The map $v: T O P(\partial M) \rightarrow \mathcal{G E}_{\partial M}(\mathcal{N}(\partial M), M)$ is a homotopy equivalence. 
Proof. The map $v=\gamma \circ \beta$ is indicated in the following diagram:

$$
T O P(\partial M) \stackrel{\beta}{\rightarrow} \mathcal{G E}_{\partial M}(\mathcal{N}(\partial M), \partial M \times[0,1)) \stackrel{\alpha}{\longrightarrow} \mathcal{G E}_{\partial M}(\mathcal{N}(\partial M), M),
$$

where $\gamma$ is the homotopy equivalence in Lemma $3.7, \beta$ is defined by $\beta=-\times i d_{[0,1)}$, and $\alpha \circ \beta=i d_{T O P(\partial M)}$. Then $\beta$ and $\alpha$ are homotopy equivalences.

Remark 3.11. The commutativity of square II follows by inspection, where the maps $v$ and $j$ are homotopy equivalences (by using the same choice of a collar), the map $u$ is the restriction map and the map $g=-\times i d_{\mathbb{R}}$.

Remark 3.12. Clearly, the map $i: \operatorname{TOP}(M \operatorname{rel} \mathcal{N}(\partial M)) \rightarrow$ TOP (Int $M$ rel $\infty)$ is an isomorphism.

Notice that the map $f: T O P(M) \rightarrow T O P($ Int $M)$ in the diagram (**) is not necessarily a fibration. Consider the following example.

Example. Let $M$ be the cylinder $S^{1} \times[0,1]$. There is a homeomorphism $\tau$ : Int $M \rightarrow$ Int $M$ that is not a restriction of a self-homeomorphism of $M$. However, $\tau$ is isotopic to the restriction of a self-homeomorphism of $M$. In other words, the map induced by the restriction $r: T O P(M) \rightarrow T O P($ Int $M)$ is not a Kan fibration.

Represent the point $x \in S^{1} \times(0, \infty)$ by $x=\left(e^{i \theta}, t\right)$, where $\theta \in[0,2 \pi)$ and $t \in(0, \infty)$.

Let $\sigma:(0, \infty) \rightarrow(0,1)$ be any homeomorphism, and for any $s \in[0,1]$ let $\rho_{s}: S^{1} \times(0, \infty) \rightarrow S^{1} \times(0, \infty)$ be a family of homeomorphisms defined by $\rho_{s}\left(e^{i \theta}, t\right)=$ $\left(e^{i(\theta+2 \pi t s)}, t\right)$. Then $\tau_{s}: S^{1} \times(0,1) \rightarrow S^{1} \times(0,1)$, defined by $\tau_{s}=(i d \times \sigma) \circ \rho_{s} \circ$ $\left(i d \times \sigma^{-1}\right)$, is an isotopy from $\rho_{s}$ to $i d$. For $s=1, \tau_{1}$ is not a restriction of any homeomorphism from $S^{1} \times[0,1]$ into itself because the image of the sequence $a_{n}=\left(e^{i \theta_{0}}, 1-1 / n\right)$ for any fixed $\theta_{0} \in[0,2 \pi)$ by $\tau_{1}$ does not converge.

\section{Wrapping homeOMORPHISMS AROUND A CIRCLE}

Let $W$ be a manifold without boundary of dimension $\geq 5$.

Theorem $\mathbf{C}$ (Wrapping homeomorphism around a circle). Let $q_{0}: W \rightarrow \mathbb{R}$ be a manifold approximate fibration. Then:

(1) There exists a manifold approximate fibration $q: \hat{W} \rightarrow S^{1}$ such that the following diagram commutes:

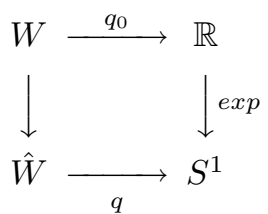

(2) $\pi_{n} T O P^{e p}(W)$ is a direct summand of $\pi_{n} \operatorname{TOP}(\hat{W})$, for $n>1$, where $\hat{W}$ is a compact and connected manifold and $W$ is the infinite cyclic cover of $\hat{W}$.

Before proving this theorem we will give some definitions.

For any topological manifold $B$, let $M A F(B)$ be the simplicial set of manifold approximate fibrations over $B$ (see [14, page 12]). If $B=S^{1}$, then a vertex of $M A F\left(S^{1}\right)$ is $q: \hat{W} \rightarrow S^{1}$, and if $B=\mathbb{R}$, a vertex of $M A F(\mathbb{R})$ is $q_{0}: W \rightarrow \mathbb{R}$.

Let $\iota: \mathbb{R} \hookrightarrow S^{1}$ be an orientation preserving embedding. Then the map $q_{\mid}$: $q^{-1}(\iota(\mathbb{R})) \rightarrow \mathbb{R}$ is a manifold approximate fibration, called the fiber germ of $q$ over $\iota$. We say that $q$ has fiber germ $q_{0}$ if and only if there exists a controlled 
homeomorphism between a manifold approximate fibration $q_{0}: W \rightarrow \mathbb{R}$ and $q_{\mid}$. See [14]. Then $\iota$ induces a map $\iota^{*}: M A F\left(S^{1}\right) \rightarrow M A F(\mathbb{R})$ which sends a manifold approximate fibration $\hat{W} \rightarrow S^{1}$ to a manifold approximate fibration $W \rightarrow \mathbb{R}$. We shall prove Theorem $\mathrm{C}$ by given a homotopy left inverse for $\iota^{*}$.

By [14, Theorem 1.4] we have the following commutative diagram:

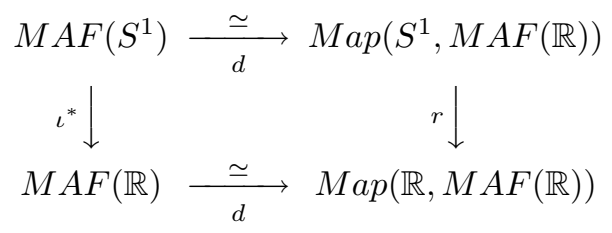

The maps $\iota^{*}$ and $r$ are the restriction maps induced by $\iota$. In order to give a left inverse to $\iota^{*}$, we construct a left inverse to $r$ which determines a left inverse to $\iota^{*}$.

Lemma 4.1. The restriction map $r: \operatorname{Map}\left(S^{1}, M A F(\mathbb{R})\right) \rightarrow \operatorname{Map}(\mathbb{R}, M A F(\mathbb{R}))$ has a homotopy left inverse.

Proof. Let $f \in \operatorname{Map}\left(S^{1}, M A F(\mathbb{R})\right)$. Then the map $r$, induced by $\iota$, is such that $r(f)=f \circ \iota \in \operatorname{Map}(\mathbb{R}, M A F(\mathbb{R}))$. Let $* \in S^{1}$. Define the restriction map $r_{\mid}: \operatorname{Map}\left(S^{1}, M A F(\mathbb{R})\right) \rightarrow \operatorname{Map}(*, M A F(\mathbb{R}))$ such that $r_{\mid}(f)=f_{\left.\right|_{*}}: * \rightarrow M A F(\mathbb{R})$ and $*$ goes to $f(*) . \quad r_{\mid}$has a homotopy left inverse $s: \operatorname{Map}(*, M A F(\mathbb{R})) \rightarrow$ $\operatorname{Map}\left(S^{1}, M A F(\mathbb{R})\right)$ defined as follows. Let $x \in M a p(*, M A F(\mathbb{R}))$. So, $x$ is a $\operatorname{map} x: * \rightarrow M A F(\mathbb{R}) ; \quad * \mapsto g$. Thus the map $s: M a p(*, M A F(\mathbb{R})) \rightarrow$ $\operatorname{Map}\left(S^{1}, M A F(\mathbb{R})\right)$ is such that $x \mapsto c_{x}$, where $c_{x}$ is the constant map, $c_{x}(z)=g$. Then, $r \circ s: M a p(*, M A F(\mathbb{R})) \rightarrow \operatorname{Map}(*, M A F(\mathbb{R}))$ is the identity. Thus, applying any isomorphism $\operatorname{Map}(\mathbb{R}, M A F(\mathbb{R})) \cong \operatorname{Map}(*, M A F(\mathbb{R}))$ which sends $0 \in \mathbb{R}$ to $*$, we have that $s$ is a homotopy left inverse of $r$. Since $\iota^{*}$ preserves base point, so do $r$ and $s$.

Proof of Theorem $C$ (1). From Lemma 4.1 and diagram $\left(^{* * *}\right), s$ determines (up to homotopy) a homotopy left inverse to $\iota^{*}$.

Thus, given any manifold approximate fibration $q_{0}: W \rightarrow \mathbb{R}$, there exists a manifold approximate fibration $\hat{q}: \hat{W} \rightarrow S^{1}$ such that with an orientation preserving embedding $\iota, q^{\prime}: W^{\prime} \rightarrow \mathbb{R}$ is controlled homeomorphic to $q_{0}: W \rightarrow \mathbb{R}$. In fact, consider the infinite cyclic cover of $\hat{W}$ and $S^{1}$. Form the pullback

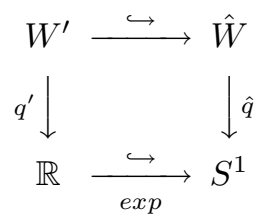

Then

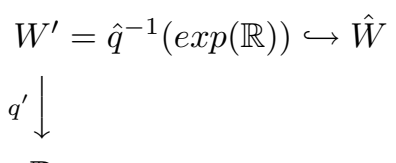

is a manifold approximate fibration (by Corollary 12.14 in [14]), and $q^{\prime}=\hat{q}_{\mid}$is fiber germ of $\hat{q}$ over exp. 
By Corollary 12.14 in [14] we have a manifold approximate fibration $q^{\prime}: W^{\prime} \rightarrow \mathbb{R}$. From the uniqueness of fiber germs [14], any two fiber germs of a manifold approximate fibration over a connected oriented manifold are controlled homeomorphic. So it follows that $q^{\prime}$ is controlled homeomorphic to $q_{0}$.

Let $M A F\left(S^{1}\right)_{q}$ denote the component of $M A F\left(S^{1}\right)$ containing $q$, and let $M A F(\mathbb{R})_{q_{0}}$ denote the component of $M A F(\mathbb{R})$ containing $q_{0}$.

By [14, Corollary 7.12] we have a commutative diagram

$(* * * *)$

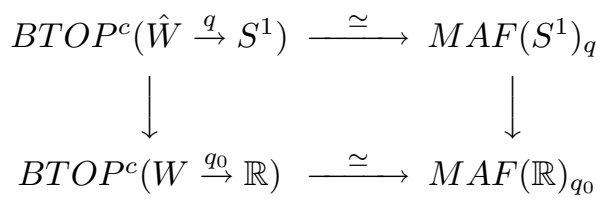

where the horizontal maps are homotopy equivalences.

Proof of Theorem C(2). From Lemma 4.1, diagram (***) and diagram (****) we have that the map $\iota_{\mid}: B T O P^{c}\left(\hat{W} \stackrel{q}{\rightarrow} S^{1}\right) \rightarrow B T O P^{c}\left(W \stackrel{q_{0}}{\rightarrow} \mathbb{R}\right)$, induced by $\iota^{*}$, has a homotopy left inverse $s_{\mid}: \operatorname{BTOP}^{c}\left(W \stackrel{q_{0}}{\rightarrow} \mathbb{R}\right) \rightarrow B T O P^{c}\left(\hat{W} \stackrel{q}{\rightarrow} S^{1}\right)$, induced by the left inverse of $\iota^{*}$. The maps $\iota_{\mid}$and $s_{\mid}$preserve base points. Thus $\iota_{\mid} \circ s_{\mid} \simeq i d$ implies that $\pi_{i}\left(\operatorname{TOP}^{c}\left(W \stackrel{q_{0}}{\rightarrow} \mathbb{R}\right)\right)$ is a direct summand of $\pi_{i}\left(\operatorname{TOP}^{c}\left(\hat{W} \stackrel{q}{\rightarrow} S^{1}\right)\right)$.

By [17, Theorem 1.1], where $B=S^{1}$, the forget control map

$$
\phi: T O P^{c}\left(\hat{W} \stackrel{q}{\rightarrow} S^{1}\right) \rightarrow T O P^{h}\left(\hat{W} \stackrel{q}{\rightarrow} S^{1}\right)
$$

is a homotopy split injective, where $\operatorname{TOP}^{h}\left(\hat{W} \stackrel{q}{\rightarrow} S^{1}\right)$ denotes the homotopy fiber of the simplicial map $\Psi: \operatorname{TOP}(\hat{W}) \rightarrow \operatorname{Map}\left(\hat{W}, S^{1}\right)$ defined by $\Psi(h)=q \circ h$, where the homeomorphism $h: \hat{W} \rightarrow \hat{W}$ is a vertex of $\operatorname{TOP}(\hat{W})$ and $\operatorname{Map}\left(\hat{W}, S^{1}\right)$ denotes the simplicial set of maps from $\hat{W}$ to $S^{1}$. Hence, a vertex of $\operatorname{TOP}^{h}\left(\hat{W} \stackrel{q}{\rightarrow} S^{1}\right)$ consists of a homeomorphism $h: \hat{W} \rightarrow \hat{W}$ together with a homotopy from $q \circ h$ to $q$. The elements of $\operatorname{TOP}^{h}\left(\hat{W} \stackrel{q}{\rightarrow} S^{1}\right)$ are called homotopically controlled. Thus, $\pi_{i}\left(T O P^{c}\left(\hat{W} \stackrel{q}{\rightarrow} S^{1}\right)\right)$ is a direct summand of $\pi_{i}\left(T O P^{h}\left(\hat{W} \stackrel{q}{\rightarrow} S^{1}\right)\right)$.

From the fibration sequence $\operatorname{TOP}^{h}\left(\hat{W} \stackrel{q}{\rightarrow} S^{1}\right) \rightarrow \operatorname{TOP}(\hat{W}) \rightarrow \operatorname{Map}\left(\hat{W}, S^{1}\right)$ we have the long exact sequence in homotopy

$$
\cdots \rightarrow \pi_{i} \operatorname{TOP}^{h}\left(\hat{W} \stackrel{q}{\rightarrow} S^{1}\right) \rightarrow \pi_{i} \operatorname{TOP}(\hat{W}) \rightarrow \pi_{i} \operatorname{Map}\left(\hat{W}, S^{1}\right) \rightarrow \cdots .
$$

With the fibration exp $: \mathbb{R} \rightarrow S^{1}$, when $\hat{W}$ is a CW complex, then the map $\operatorname{Map}(\hat{W}, \mathbb{R}) \rightarrow \operatorname{Map}\left(\hat{W}, S^{1}\right)$ is a fibration. Since $\mathbb{R} \simeq *$, we have $\operatorname{Map}(\hat{W}, \mathbb{R}) \simeq$ $\operatorname{Map}(\hat{W}, *) \simeq *$. Thus, $* \simeq \operatorname{Map}(\hat{W}, \mathbb{R}) \rightarrow \operatorname{Map}\left(\hat{W}, S^{1}\right)_{\text {certain components (i.e. }}$ components of the homotopy trivial map) implies $\pi_{i} \operatorname{Map}\left(\hat{W}, S^{1}\right)_{*}=0$, for $i>1$. Thus, $\pi_{i} T O P^{h}\left(\hat{W} \stackrel{q}{\rightarrow} S^{1}\right) \cong \pi_{i} \operatorname{TOP}(\hat{W})$, for $i>1$.

So, $\pi_{i} T O P^{c}\left(W \stackrel{q_{0}}{\rightarrow} \mathbb{R}\right)$ is a direct summand of $\pi_{i} T O P^{c}\left(\hat{W} \stackrel{q}{\rightarrow} S^{1}\right)$; likewise $\pi_{i} T O P^{c}\left(\hat{W} \stackrel{q}{\rightarrow} S^{1}\right)$ is a direct summand of $\pi_{i} T O P^{h}\left(\hat{W} \stackrel{q_{0}}{\rightarrow} S^{1}\right)$, and by Theorem $1.11, \pi_{i} T O P^{c}\left(W \stackrel{q_{0}}{\rightarrow} \mathbb{R}\right) \cong \pi_{i} T O P^{e p}(W)$.

Since $W$ is the infinite cyclic cover of $\hat{W}$ induced by $q: \hat{W} \rightarrow S^{1}$ from $\exp : \mathbb{R} \rightarrow$ $S^{1}$, the map $p: W \hookrightarrow \hat{W}$ induces a map $T O P(\hat{W}) \rightarrow T O P^{e p}(W)$.

Hence, for $i>1, \pi_{i} T O P^{e p}(W)$ is a direct summand of $\pi_{i} \operatorname{TOP}(\hat{W})$.

Lemma 4.2. $\pi_{1} \operatorname{Map}\left(\hat{W}, S^{1}\right) \simeq \mathbb{Z}$ and $\pi_{0} \operatorname{Map}\left(\hat{W}, S^{1}\right) \simeq H^{1}(\hat{W}, \mathbb{Z})$. 
Proof. Let $\hat{W}$ be a connected compact manifold and consider the fibration sequence $\mathbb{Z} \hookrightarrow \mathbb{R} \stackrel{\exp }{\rightarrow} S^{1}$. Then $\operatorname{Map}\left(\hat{W}, S^{1}\right)$ is a fibration and since $\mathbb{R} \simeq *, \operatorname{Map}(\hat{W}, \mathbb{R}) \simeq$ $\operatorname{Map}(\hat{W}, *) \simeq *$, which implies $\pi_{i} \operatorname{Map}\left(\hat{W}, S^{1}\right)_{*}=0$, for $i>1$. From the fibration sequence $\operatorname{Map}(\hat{W}, \mathbb{Z}) \hookrightarrow \operatorname{Map}(\hat{W}, \mathbb{R}) \rightarrow \operatorname{Map}\left(\hat{W}, S^{1}\right)$ we have a exact sequence in homotopy

$$
\cdots \rightarrow 0 \rightarrow \pi_{1} \operatorname{Map}\left(\hat{W}, S^{1}\right) \rightarrow \pi_{0} \operatorname{Map}(\hat{W}, \mathbb{Z}) \rightarrow 0 \rightarrow \cdots
$$

which implies $\pi_{1} \operatorname{Map}\left(\hat{W}, S^{1}\right)_{*} \cong \operatorname{Map}(\hat{W}, \mathbb{Z}) \simeq \mathbb{Z}$.

Now, $S^{1}$ is a topological group, so $\operatorname{Map}\left(\hat{W}, S^{1}\right)$ is an $H$-space, which implies any two path components of $\operatorname{Map}\left(\hat{W}, S^{1}\right)$ are homotopy equivalent. Thus,

$$
\pi_{0} \operatorname{Map}\left(\hat{W}, S^{1}\right)=\left[\hat{W}, S^{1}\right]=[\hat{W}, K(\mathbb{Z}, 1)]=H^{1}(\hat{W}, \mathbb{Z}) .
$$

Conclusion: If $\hat{W}$ is a connected, compact manifold, then $\operatorname{Map}\left(\hat{W}, S^{1}\right) \stackrel{\text { weak }}{\simeq}$ $H^{1}(\hat{W}, \mathbb{Z}) \times S^{1}$.

Remark 4.3. By Lemma 4.2, $\pi_{1} \operatorname{Map}\left(\hat{W}, S^{1}\right) \simeq \mathbb{Z}$ and $\pi_{0} \operatorname{Map}\left(\hat{W}, S^{1}\right) \simeq H^{1}(\hat{W}, \mathbb{Z})$. Thus,

$$
\cdots \rightarrow 0 \rightarrow \pi_{1} T O P^{h}\left(\hat{W} \stackrel{q}{\rightarrow} S^{1}\right)>\pi_{1} T O P(\hat{W}) \rightarrow \mathbb{Z} \rightarrow \cdots .
$$

And hence,

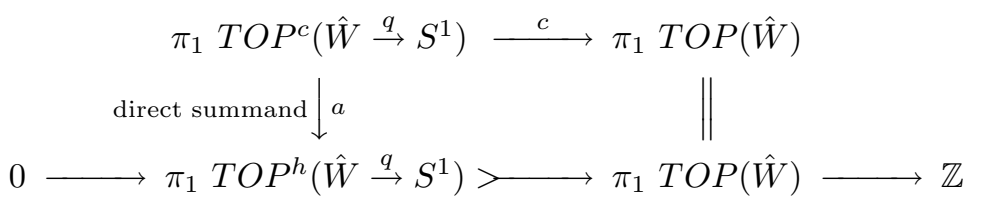

So, $c$ is injective.

\section{REFERENCES}

1. J. Adams, On the triad connectivity theorem, unpublished lecture notes.

2. D. Anderson and W. Hsiang, Extending combinatorial PL structures on stratified spaces I, Inv. Math. 32 (1976), 179-204; II, Trans. Amer. Math. Soc. 260 (1980), 223-253. MR 54:1235; MR 81h:57009

3. 201-223. MR 55:13447

4. D. Burghelea, Automorphisms of manifolds, Proc. Symp. Pure Math. 32 part 1 (1978), 347371. MR 80g:57001

5. D. Burghelea, R. Lashof and M. Rothenberg, Groups of automorphisms of manifolds, vol. 473, Lecture Notes in Math., Spring-Verlag, NY, 1975. MR 52:1738

6. T. A. Chapman, Approximation results in topological manifolds, Memoirs of the AMS 34 (1981), no. 257. MR 83i:57005

7. R. Edwards and R. Kirby, Deformations of spaces of embeddings, Ann. of Math. 93 (1971), 63-88. MR 44:1032

8. M. Freedman and F. Quinn, Topology of 4-manifolds, Princeton University, Princeton, New Jersey, 1990. MR 94b:57021

9. A. Hatcher, Concordance spaces, higher simple homotopy theory and applications, Proc. Symp. Pure Math. 32 part 1 (1978), 3-23. MR 80f:57014

10. Homotopy Theory (E. Rees and J. D. S. Jones, editors), Proc. Durham Symp. London Math. Soc. Lect. Note Series 117 (1985). MR 88j:55002

11. W.-C. Hsiang, Geometric applications of algebraic K-theory, In Proc. ICM, PWN, Warsaw (1983), pp. 99-118. MR 87g:57034 
12. B. Hughes, Approximate fibrations on topological manifolds, Michigan Math. J. 32 (1985), 167-183. MR 87e:57025

13. B. Hughes and A. Ranicki, Ends of complexes, Cambridge Univ. Press, 1996. MR 98f:57039

14. B. Hughes, L. Taylor and B. Williams, Bundles theories for topological manifolds, Trans. of AMS 319 (1990), 1-65. MR 91e:57035

15. Manifold approximate fibrations are approximately bundles, Forum Math. 3 (1991), 309-325. MR 92k:57040

16. _ Bounded homeomorphisms over Hadamard manifolds, Math. Scand. 73 (1993), 161176. MR 95h:57042

17. Splitting Forget Control Maps, in preparation.

18. B. Hughes, L. Taylor, S. Weinberger and B. Williams, Neighborhoods in Stratified Spaces. I. Two Strata (to appear).

19. K. Igusa, Parametrized Morse Theory and its Applications, Proc. ICM, Kyoto, Japan (1990), 643-651. MR 93c:57022

20. R. Kirby, Stable homeomorphisms and the annulus conjecture, Ann. of Math. 89 (1969), 575-582. MR 39:3499

21. R. Kirby and L. Siebenmann, Foundational essays on topological manifolds, smoothings, and triangulations, vol. 88, Ann. of Math. Studies, Princeton University Press, 1977. MR 58:31082

22. J. Kister, Microbundles are fibre bundles, Ann. of Math. 80 (1964), 190-199. MR 31:5216

23. N. Kuiper and R. Lashof, Microbundles and bundles: I, Invent. Math. 1 (1966), 1-17; II, Invent. Math. 1 (1966), 243-259. MR 35:7339; MR 35:7340

24. R. Lashof and M. Rothenberg, G-smoothing theory, Proc. of Symposia in Pure Math. 32 (1978), 211-266. MR 80h:57030

25. A. Nicas, Induction theorems for groups of homotopy manifold structures, Memoirs of AMS 39 (1982), no. 267. MR 83i:57026

26. F. Quinn, Ends of maps - I, Ann. of Math. 110 (1979), 275-331. MR 82k:57009

27. Homotopically stratified sets, J. Amer. Math. Soc. 1 (1988), 441-499. MR 89g:57050

28. C. Rourke and B. Sanderson, Introduction to PL Topology, Spring-Verlag, New York, 1972. MR 50:3236

29. T. B. Rushing, Topological embeddings, Academic Press, New York, 1973. MR 50:1247

30. L.Siebenmann, The obstruction to finding a boundary for an open neighborhood, Ph.D. Thesis, Princeton University, Princeton, 1966.

31. _ The structure of tame ends, Notices of AMS 13 (1966), 862.

32. - A torsion invariant for bands, Notices of AMS 15 (1968), 811.

33. A total Whitehead torsion obstruction to fibering over the circle, Comment. Math. Helv. 45 (1970), 1-48. MR 44:4768

34. _ Deformation of homeomorphisms on stratified sets. I, II, Comment. Math. Helv. 47 (1972), 123-163. MR 47:7752

35. Regular (or canonical) open neighborhoods, General Topology and its Applications 3 (1973), 51-61. MR 51:6831

36. L.Siebenmann, L. Guillou and H. Hähl, Les Voisinages Ouverts Réguliers, Ann. Sci. E.N.S. (1973), 253-293. MR 48:9732

37. Les voisinages ouverts réguliers: critères homotopiques d'existence, Ann. Sci. E.N.S. (1974), 431-462. MR 50:14766

38. M. Weiss and B. Williams, Automorphisms of Manifolds and Algebraic K-Theory I, K-Theory 1 (1988), 575-626. MR 89h:57012

Univeridade Federal Fluminense - UFF, Instituto de Matemática, Departamento de AnÁlise, Niterói, RJ, 24020-005 - Brazil

E-mail address: ganancy@vm.uff.br 